\title{
Assessing cerebrovascular autoregulation in infants with necrotizing enterocolitis using near-infrared spectroscopy
}

\author{
Trijntje E. Schat ${ }^{1}$, Michelle E. van der Laan', Maarten Schurink², Jan B.F. Hulscher², Christian V. Hulzebos' ${ }^{1}$, Arend F. Bos ${ }^{1}$ \\ and Elisabeth M.W. Kooi'
}

BACKGROUND: We assessed cerebrovascular autoregulation (CAR) in preterm infants with definite necrotizing enterocolitis (NEC), Bell's stage 2 or 3 , and infants without NEC, using near-infrared spectroscopy. We hypothesized that CAR would be more often impaired in infants with NEC compared with infants without NEC.

METHODS: We measured cerebral regional tissue oxygen saturation, arterial oxygen saturation, and mean arterial blood pressure (MABP) during $48 \mathrm{~h}$. We calculated the correlation between cerebral fractional tissue oxygen extraction and MABP for each patient. A statistically significant negative correlation reflected impaired CAR.

RESULTS: We included 15 infants with definite NEC (median (range) gestational age 27.4 (25.6-34.7) wk; birth weight 1,070 $(670-2,400) \mathrm{g})$ and 13 infants without NEC (gestational age 27.9 (26.3-34.7) wk; birth weight $980(640-2,640) \mathrm{g})$. Fourteen infants had a statistically significant negative correlation ( $\rho-0.468$ to- 0.104$)$, of whom five were infants without NEC $(5 / 13 ; 38 \%)$ and nine with definite NEC $(9 / 15 ; 60 \%)$. The difference in prevalence of impaired CAR was not statistically significant.

CONCLUSION: Impaired CAR is present in a substantial proportion of infants with definite NEC, which may predispose them to NEC-associated neurological damage.

N ecrotizing enterocolitis (NEC) is a devastating gastrointestinal disease that predominantly affects preterm infants (1). Several studies demonstrated a strong association between NEC and impaired neurodevelopmental outcome (2-7). Moreover, neurodevelopmental delay in preterm infants with NEC assessed at school age was found to be associated with more white matter abnormalities as seen on magnetic resonance imaging at term compared to infants who were also born prematurely but who did not have NEC (8). White matter abnormalities are considered to be the principal anatomic substrate for the neurodevelopmental disability in infants with NEC (9).

The development of white matter injury depends on the occurrence of two separate mechanisms: infection and/or inflammation and ischemia (10). Infants with NEC develop an excessive inflammatory response with production of inflammatory toxins (11). This may affect systemic circulation, and-if severe-also cerebral circulation. Ischemia of cerebral tissue is the result of diminished cerebral blood flow (CBF) caused, among others, by impaired cerebrovascular autoregulation (CAR). CAR plays a pivotal role in regulating CBF. When CAR is affected, a pressure-passive cerebral circulation arises, i.e., changes in blood pressure cause changes in CBF. When CBF is low, underperfusion of cerebral tissue ensues, which may lead to cerebral ischemia and subsequent brain damage.

Our research group demonstrated that near-infrared spectroscopy (NIRS) can be used to estimate the presence or absence of CAR in otherwise stable preterm infants, by assessing the relationship between mean arterial blood pressure (MABP) and cerebral fractional tissue oxygen extraction (FTOE). We assumed that a statistically significant negative correlation between MABP and cerebral FTOE reflects impaired CAR. CAR was found to be impaired in $40 \%$ of relatively stable preterm infants, also in infants for whom blood pressures were in the "normal" range (12). In the absence of CAR, the relationship between MABP and cerebral perfusion is linear. Because FTOE is a reflection of cerebral perfusion, changes in MABP cause opposite changes in cerebral FTOE if CAR is impaired. This linear relationship is independent of the values measured and, as such, can also occur in the presence of blood pressures in the "normal" range.

It is unknown what the prevalence is of impaired CAR in preterm infants with NEC and whether CAR is impaired more often in preterm infants with established NEC than in infants without NEC. Our aim was, therefore, to assess cerebral hemodynamics using NIRS in preterm infants with NEC (Bell's stage 2 or 3 ) and without NEC and to compare the prevalence of impaired CAR between these two groups. We hypothesized that definite NEC would be associated with a higher prevalence of impaired CAR in comparison to infants without NEC, due to the presence of infection/inflammation leading to an affected cerebral circulation.

\footnotetext{
'Division of Neonatology, Beatrix Children's Hospital, University Medical Center Groningen, University of Groningen, Groningen, The Netherlands; ${ }^{2}$ Division of Pediatric Surgery, Department of Surgery, University Medical Center Groningen, University of Groningen, Groningen, The Netherlands. Correspondence: Trijntje E. Schat (t.e.schat@alumnus.rug.nl)

Received 9 December 2014; accepted 15 July 2015; advance online publication 21 October 2015. doi:10.1038/pr.2015.184
} 
RESULTS

We included 15 infants with definite NEC and 13 control infants. We present the patient characteristics for the two groups in Table 1. Infants with NEC had significantly higher $\mathrm{PCO}_{2}$ and C-reactive protein concentrations. NIRS monitoring in infants with NEC had started significantly later compared with the control group. Furthermore, infants with definite NEC received inotropes, volume expansion, and red blood cell transfusions significantly more often during the study period compared with controls. With regard to inotropes administration, three infants received dopamine only, two infants dobutamine, and two infants both dopamine and dobutamine.

Of the six infants with NEC who died, four infants did so as a result of circulatory and respiratory insufficiency within the 48-h study period. The fifth infant died due to circulatory insufficiency $10 \mathrm{~d}$ after the NIRS measurements-most likely as a result of an anastomotic leak. The sixth infant died $89 \mathrm{~d}$ after the study period due to irreversible brain damage, which most likely occurred during surgery for an intestinal obstruction. The infant without NEC who died did so $66 \mathrm{~d}$ after the study period, due to bronchopulmonary dysplasia.

In the infants with NEC, NIRS recordings started after a median time of $11 \mathrm{~h}$ (range: 2-29h) after onset of NEC symptoms and were discontinued preliminary in six infants due to the presence of pneumoperitoneum on abdominal radiographic examination.

Out of the 15 infants in the NEC group, we found 9 (60\%) with a statistically significant negative correlation between MABP and cerebral FTOE. Out of the 13 infants without NEC, we found 5 infants (38\%) with a statistically significant negative correlation. This difference was not statistically significant

Table 1. Patient characteristics of infants without NEC and infants with definite NEC

\begin{tabular}{lcc}
\hline & No NEC $(n=13)$ & Definite NEC $(n=15)$ \\
\hline Gestational age (weeks) & $27.9(26.3-34.7)$ & $27.4(25.6-34.7)$ \\
Birth weight (grams) & $980(640-2,640)$ & $1,070(670-2,400)$ \\
Postnatal age (days) & $4(3-13)$ & $10(3-34)^{*}$ \\
Male/female & $6 / 7$ & $10 / 5$ \\
Bell's stage 2/Bell's stage 3 & $\mathrm{NA}$ & $5 / 10$ \\
Hemoglobin (mmol/l) & $8.3(7.3-10.5)(n=10)$ & $7.4(6.0-12.0)$ \\
PCO (kPa) & $5.6(4.8-6.6)(n=10)$ & $6.5(4.4-10.1)^{*}$ \\
Glucose (mmol/l) & $4(3.2-7)(n=7)$ & $6.5(3.2-8.6)$ \\
CRP (mg/l) & $0(0-8)(n=7)$ & $110(7-425)^{* *}$ \\
Mechanical ventilation (\%) & $6(46)$ & $12(80)$ \\
hsPDA (\%) & $4(31)$ & $1(7)$ \\
Inotropes (\%) & $0(0)$ & $7(47)^{*}$ \\
Volume expansion (\%) & $1(8)$ & $12(80)^{* *}$ \\
RBC transfusion (\%) & $1(8)$ & $8(53)^{*}$ \\
Mortality (\%) & $1(8)$ & $6(40)$
\end{tabular}

Data are expressed as median (range) or as numbers unless specified otherwise. Statistical differences between the two groups are marked by* $(<0.05)$ or ${ }^{* *}(<0.001)$. CRP, C-reactive protein; hsPDA, hemodynamically significant patent ductus arteriosus; NA, not applicable; NEC, necrotizing enterocolitis; RBC, red blood cell.
$(P=0.256)$. Table 2 shows the correlation coefficients with associated $P$ values per infant.

In Figure 1, we present the correlation coefficients between cerebral FTOE and MABP for one infant without NEC without a statistically significant negative correlation suggesting adequate CAR and for one infant with NEC with a statistically significant negative correlation suggesting impaired CAR. The infant with definite NEC was monitored for $8 \mathrm{~h}$ until pneumoperitoneum was detected on abdominal radiographic examination. The infant was taken to theater shortly afterwards.

\section{DISCUSSION}

We demonstrated that $60 \%$ of preterm infants with definite NEC (Bell's stage 2 or 3 ) had a statistically significant negative correlation between cerebral FTOE and MABP, suggesting impaired CAR. This is a high percentage in comparison to the preterm infants without NEC (38\%). Nevertheless, this difference was not statistically significant.

For preterm infants who suffered NEC, the short-term and long-term neurological sequelae can be devastating. Timely identification of infants with impaired CAR is, therefore, important in order to prevent the development of white matter abnormalities. Our data indicate that, to this end, NIRS is a useful, noninvasive, bedside monitoring method.

Several studies investigated the presence of CAR in preterm infants using NIRS (12-17). The prevalence of pressure passivity ranged from 14 to $53 \%$. These studies, however, all used different parameters to assess CAR and also performed their analyses differently, a fact which complicates any comparison. Our definition of impaired CAR was based on a study performed previously in our research group. Verhagen et al. (12) demonstrated that $40 \%$ of clinically stable preterm infants had a statistically significant negative correlation between cerebral FTOE and MABP. We found similar results in our control group.

In preterm infants with definite NEC, we found a statistically significant negative correlation, which suggested impaired CAR, between cerebral FTOE and MABP in $60 \%$ of cases. A possible explanation for this higher percentage could be that preterm infants with NEC might have had blood pressures under the lower limit of the autoregulatory curve, with a consequential pressure-passive CBF. We did not find statistically significant negative correlations between cerebral FTOE and MABP for those infants who had the lowest mean MABP. Indeed, it was suggested that impaired CAR might develop when blood pressures are in the range of what we now define as "normal" and "safe" $(12,14)$. In these instances, it is of the utmost importance to only allow minimal decreases in blood pressure in order to prevent low cerebral perfusion and the subsequent risk of developing brain injury.

Factors that were found to be associated with impaired CAR are low birth weight $(14,15)$, low gestational age (15), low hemoglobin (18), low glucose (19), and high $\mathrm{PCO}_{2}$ concentrations (20, 21). $\mathrm{PCO}_{2}$ concentrations were significantly higher in preterm infants with NEC, which suggests a possible contributing role of $\mathrm{PCO}_{2}$ in causing pressure-passive $\mathrm{CBF}$ in the preterm infants 
Table 2. Individual correlation coefficients between cerebral FTOE and MABP, sampled every 5 min

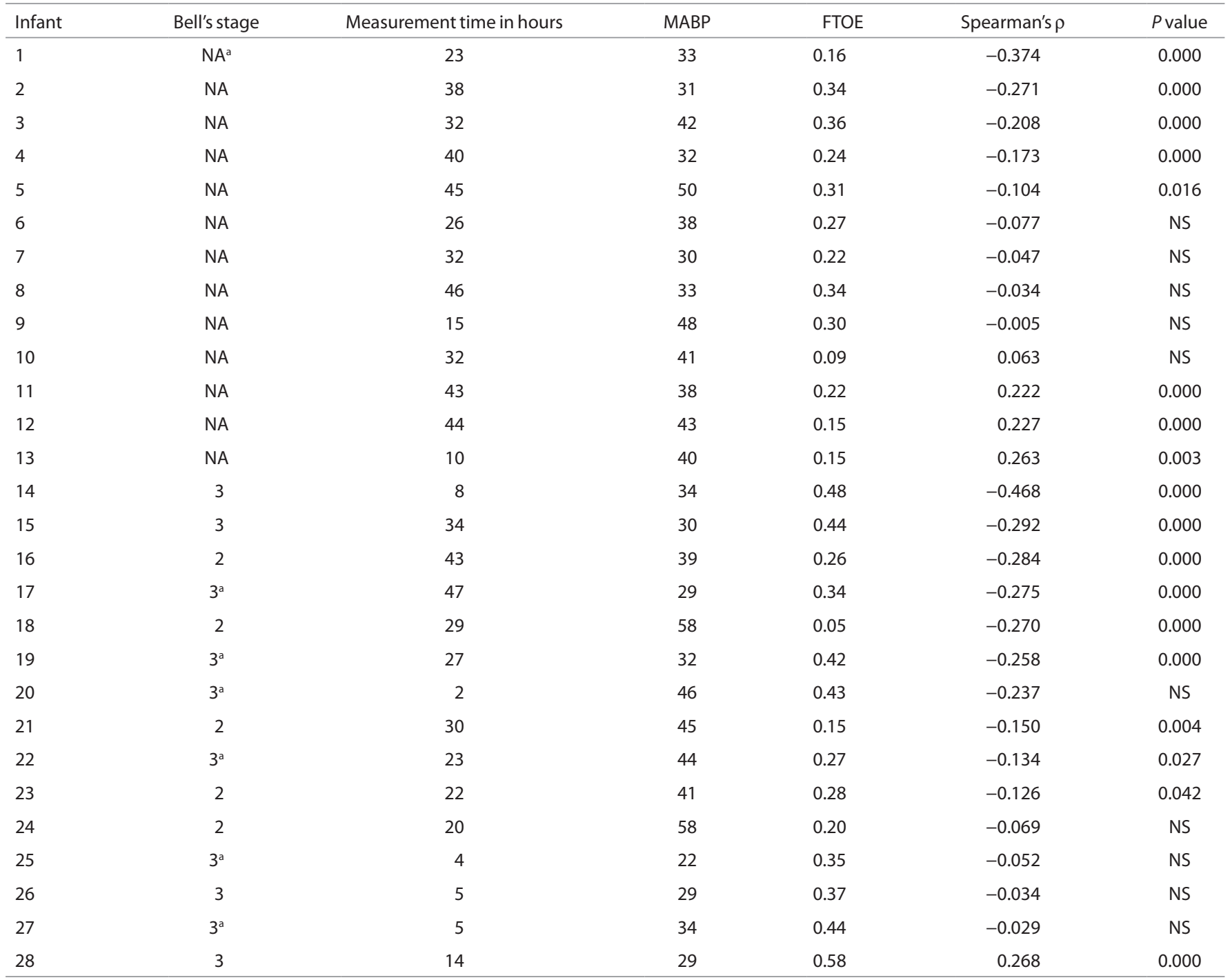

The mean values of MAPB and FTOE are presented. Correlation coefficients are based on the correlation between MABP and FTOE, sampled every 5 min. FTOE, fractional tissue oxygen extraction; MABP, mean arterial blood pressure; NA, not applicable.

anfants who died.
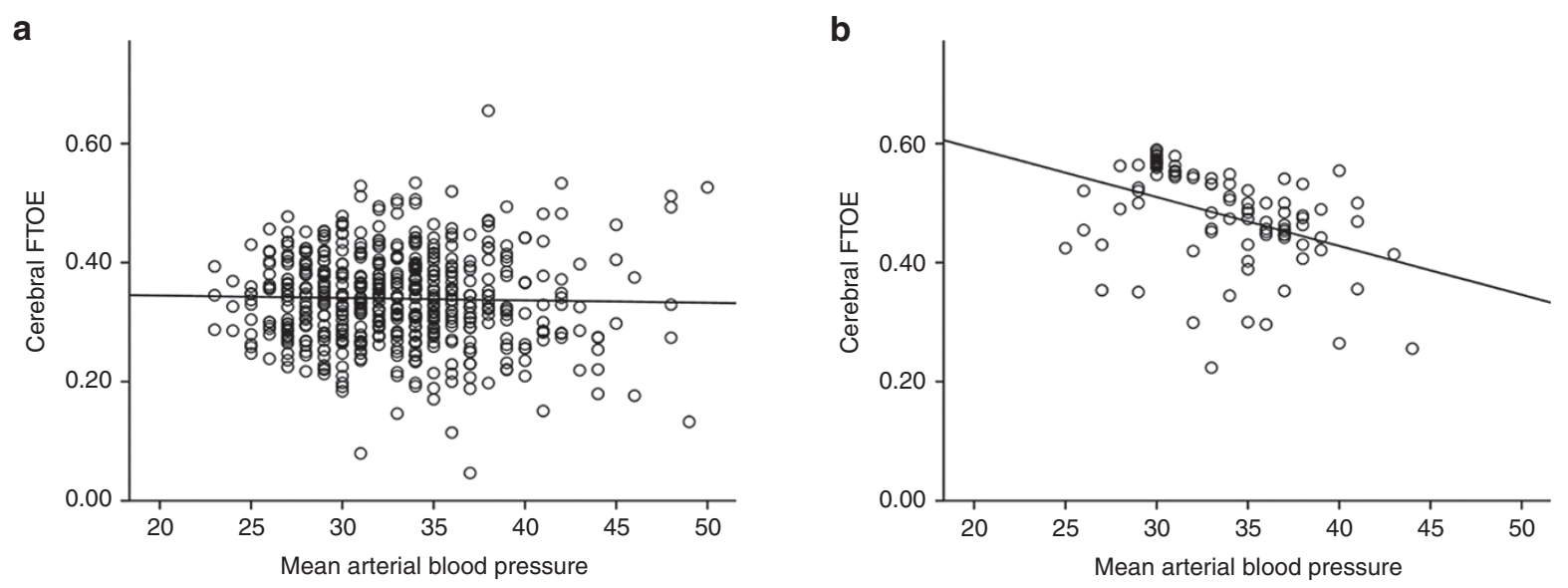

Figure 1. Scatter plot of cerebral FTOE and MABP values in an infant with (a) adequate CAR and (b) an infant with impaired CAR. CAR, cerebrovascular autoregulation; FTOE, fractional tissue oxygen extraction; MABP, mean arterial blood pressure. 
with NEC we studied. Nevertheless, we would like to stress that in our study group the $\mathrm{PCO}_{2}$ values were based on single measurements and may, therefore, not represent the entire study period. Additionally, infants with NEC received inotropes more often compared with infants without NEC. It was found that treated hypotension was associated with short- and long-time morbidity, which suggests an indirect relationship between inotropes and a pressure-passive cerebral circulation $(22,23)$. Furthermore, a direct negative influence of dopamine on CAR has been reported (24). Conversely, several studies demonstrated a protective role of inotropes by means of increasing CBF (25-27).

Limitations of this study are the small size of the sample and the variation in the duration of the cerebral regional tissue oxygen saturation $\left(\mathrm{r}_{\mathrm{C}} \mathrm{SO}_{2}\right)$ measurements for assessing CAR. These varied between 2 and $47 \mathrm{~h}$. Recent research suggested that pressure passivity can be transiently impaired (13-16). In infants whom we measured for a short period of time, we might, on the one hand, have measured an episode in which pressure passivity was present or, on the other hand, an episode in which this was not the case. It is, therefore, possible that in such instances we either underestimated or overestimated the prevalence of impaired CAR. Furthermore, we were only able to sample one value of arterial oxygen saturation $\left(\mathrm{SpO}_{2}\right)$ every $5 \mathrm{~min}$ due to technical limitations. Therefore, our cerebral FTOE is based on one single value every $5 \mathrm{~min}$, instead of a mean value over 5 -min sampling at $0.2 \mathrm{~Hz}$. Nevertheless, we have previously demonstrated that even with this relatively low sampling rate, we were able to identify infants with absence of CAR (12). Possibly, infants with statistically significant negative correlations in our study group are the infants in whom CAR was lacking during most of the study period. We may certainly have missed subtle changes in CAR, within these 5-min periods. Before NIRS can be implemented in clinical practice, the most optimal sampling rate and sampling time need to be determined. Finally, we did not control for potential confounders, such as inotropes, volume expansion, and red blood cell transfusions, which might have affected $\mathrm{CBF}$ and CAR. Future studies should investigate this further.

In conclusion, we demonstrated that $60 \%$ of preterm infants with NEC had a statistically significant negative correlation between cerebral FTOE and MABP, which may be indicative of impaired CAR. Our findings may imply that a pressure-passive CBF can be the underlying pathophysiological substrate for the development of white matter abnormalities and the long-term neurodevelopmental impairments in a substantial proportion of preterm infants with NEC. Furthermore, our results indicated that in those infants in whom NIRS measurements suggested impaired CAR, fluctuations in blood pressure could be potentially harmful. This warrants monitoring blood pressure closely as well as monitoring cerebral oxygenation by means of NIRS. Future studies need to be performed to elucidate when and how we need to intervene.

\section{METHODS}

\section{Study Population}

We included infants with definite NEC who were part of a larger, prospective observational cohort study performed in the neonatal intensive care unit of the University Medical Center Groningen, between
October 2010 and October 2012. This study was registered in the Dutch Trial Registry under number NTR3239. For the purpose of this study, we included only those preterm infants with a continuous invasive arterial blood pressure measurement who were diagnosed with definite NEC. After completion of the study, an expert panel of consultant neonatologists and pediatric surgeons independently classified the infants into the modified Bell's stages (28). Consensus was reached in all cases. We defined the onset of NEC symptoms as the time of the first abdominal radiographic examination taken after clinical suspicion of NEC, including those taken in referring hospitals.

The study was approved by the institutional ethics review board of the University Medical Center Groningen. Written parental informed consent was obtained in all cases.

We selected control infants who were admitted to our neonatal intensive care unit between October 2010 and December 2012. The attending neonatologist decided on NIRS monitoring on clinical grounds. We only included infants with a gestational age $<35 \mathrm{wk}$, with an indwelling arterial catheter for constant blood pressure measurements. Exclusion criteria were the presence of NEC or sepsis.

\section{Near-Infrared Spectroscopy}

We measured $\mathrm{r}_{\mathrm{c}} \mathrm{SO}_{2}$ continuously with a near-infrared spectrometer (INVOS 5100C, Covidien, Mansfield, MA). The neonatal Somasensor (Covidien) was placed on the frontoparietal side of the infant's head and was secured with elastic bandaging or Mepitel (Mölnlycke, Sweden).

The sensor emits two wavelengths $(730$ and $810 \mathrm{~nm})$ in the cerebral tissue. The two receivers, at 30 - and $40-\mathrm{mm}$ distance from the emitter, receive the reflected light as a function of wavelength. Hence, the spectral absorption of the underlying tissue can be determined (29,30). Since oxygenated and deoxygenated hemoglobin each absorb and reflect nearinfrared light at the two wavelengths differently, it is possible to calculate regional tissue oxygen saturation, i.e., microvascular oxygenation.

We measured $\mathrm{SpO}_{2}$ (Nellcor, Covidien) simultaneously with $\mathrm{r}_{\mathrm{c}} \mathrm{SO}_{2}$ and calculated cerebral FTOE using the formula: $\mathrm{FTOE}=\left(\mathrm{SpO}_{2}-\right.$ $\left.\mathrm{r}_{\mathrm{c}} \mathrm{SO}_{2}\right) / \mathrm{SpO}_{2}$ (ref. 31). FTOE reflects the balance between tissue oxygen supply and tissue oxygen consumption. As cerebral oxygen consumption is thought to be relatively stable in preterm infants, FTOE can be used as an indicator of tissue perfusion (31).

\section{Analysis of CAR}

A blood pressure-dependent CBF (pressure-passive CBF) can be the result of two mechanisms: blood pressures below the autoregulatory threshold or impaired CAR. Cerebral FTOE was found to be inversely related to CBF (32). In the presence of impaired CAR, FTOE will also be blood pressure dependent. In that case, low blood pressures cause higher cerebral oxygen extraction in order to meet the needs for normal cerebral metabolism. We used this negative relationship between arterial blood pressure and cerebral FTOE to assess the absence of CAR. We considered a statistically significant negative correlation coefficient as indicating impaired CAR (12).

\section{Clinical Data}

We collected the following neonatal demographic and clinical characteristics: birth weight, gestational age, postnatal age at first NIRS measurement, the concentration of hemoglobin, glucose, $\mathrm{PCO}_{2}$, and C-reactive protein, the need of mechanical ventilation, and mortality. Additionally, we documented the administration of red blood cell transfusions, volume expansion, and inotropes, and the presence of a hemodynamically significant patent ductus arteriosus during the study period. Hemodynamically significant patent ductus arteriosus was defined as a diastolic forward flow in the branches of the pulmonary artery, a diastolic backflow in the descending aorta, and a left ventricular end diastolic diameter greater than the 95th centile.

We determined hemoglobin and $\mathrm{PCO}_{2}$ as the first value just before or during the NIRS-recording period. The glucose concentration is based on the lowest value during NIRS recording, while the C-reactive protein concentration is based on the highest value during NIRS recording.

\section{Statistics}

In infants with $\mathrm{NEC}, \mathrm{rSO}_{2}$ recording started as soon as possible after clinical signs and symptoms suggested the presence of NEC. We 
sampled one random value of $\mathrm{r} \mathrm{SO}_{2}, \mathrm{SpO}_{2}, \mathrm{FTOE}$, and MABP every $5 \mathrm{~min}$ for 48 consecutive hours for both infants with NEC and control infants. Next, we calculated 48 -h mean values for each variable. Artifacts in the measurements were not taken into the analyses. The Spearman rank test was used to calculate the correlation coefficients between MABP and cerebral FTOE, sampled every $5 \mathrm{~min}$, for each preterm infant individually. Next, we compared the prevalence of impaired CAR between infants without NEC and infants with definite NEC (Bell's stage 2 or 3 ) using $\chi^{2}$ test.

We compared proportions of categorical data with Fisher exact test, and median values were analyzed using the Mann-Whitney test for nonnormal distributions. We used SPSS 22.0 software for Windows (IBM SPSS Statistics 22; IBM, Armonk, NY) for all our statistical analyses. A $P$ value of $<0.05$ was considered statistically significant.

\section{ACKNOWLEDGMENTS}

This study was part of the research program of the postgraduate school for Behavioural and Cognitive Neurosciences, University of Groningen. We greatly acknowledge the help of T. van Wulfften Palthe for correcting the English of this article.

\section{STATEMENT OF FINANCIAL SUPPORT}

This study was funded by the Research Foundation of Beatrix Children's Hospital (Groningen, The Netherlands), the Doelmatigheidsfonds (a costeffectiveness fund) of University Medical Center Groningen (Groningen, The Netherlands), and an unrestricted grant from the NutsOhra Foundation (Amsterdam, The Netherlands). T.E.S. and M.E.v.d.L. were financially supported by a grant from the Junior Scientific Master Class of the University of Groningen.

Disclosure: The authors declare that they have nothing to disclose, financially or otherwise. There is no conflict of interest.

\section{REFERENCES}

1. Sharma R, Hudak ML. A clinical perspective of necrotizing enterocolitis: past, present, and future. Clin Perinatol 2013;40:27-51.

2. Stoll BJ, Hansen NI, Adams-Chapman I, et al.; National Institute of Child Health and Human Development Neonatal Research Network. Neurodevelopmental and growth impairment among extremely low-birth-weight infants with neonatal infection. JAMA 2004;292:2357-65.

3. Hintz SR, Kendrick DE, Stoll BJ, et al.; NICHD Neonatal Research Network. Neurodevelopmental and growth outcomes of extremely low birth weight infants after necrotizing enterocolitis. Pediatrics 2005;115: 696-703.

4. Rees CM, Pierro A, Eaton S. Neurodevelopmental outcomes of neonates with medically and surgically treated necrotizing enterocolitis. Arch Dis Child Fetal Neonatal Ed 2007;92:F193-8.

5. Schulzke SM, Deshpande GC, Patole SK. Neurodevelopmental outcomes of very low-birth-weight infants with necrotizing enterocolitis: a systematic review of observational studies. Arch Pediatr Adolesc Med 2007;161: 583-90.

6. Martin CR, Dammann O, Allred EN, et al. Neurodevelopment of extremely preterm infants who had necrotizing enterocolitis with or without late bacteremia. J Pediatr 2010;157:751-6.e1.

7. Roze E, Ta BD, van der Ree MH, et al. Functional impairments at school age of children with necrotizing enterocolitis or spontaneous intestinal perforation. Pediatr Res 2011;70:619-25.

8. Shah DK, Doyle LW, Anderson PJ, et al. Adverse neurodevelopment in preterm infants with postnatal sepsis or necrotizing enterocolitis is mediated by white matter abnormalities on magnetic resonance imaging at term. J Pediatr 2008;153:170-5, 175.e1

9. Volpe JJ. Postnatal sepsis, necrotizing entercolitis, and the critical role of systemic inflammation in white matter injury in premature infants. J Pediatr 2008;153:160-3.

10. Khwaja O, Volpe JJ. Pathogenesis of cerebral white matter injury of prematurity. Arch Dis Child Fetal Neonatal Ed 2008;93:F153-61.

11. Neu J, Walker WA. Necrotizing enterocolitis. N Engl J Med 2011;364: 255-64.
12. Verhagen EA, Hummel LA, Bos AF, Kooi EM. Near-infrared spectroscopy to detect absence of cerebrovascular autoregulation in preterm infants. Clin Neurophysiol 2014;125:47-52.

13. Tsuji M, Saul JP, du Plessis A, et al. Cerebral intravascular oxygenation correlates with mean arterial pressure in critically ill premature infants. Pediatrics 2000;106:625-32.

14. Soul JS, Hammer PE, Tsuji M, et al. Fluctuating pressure-passivity is common in the cerebral circulation of sick premature infants. Pediatr Res 2007;61:467-73.

15. Wong FY, Leung TS, Austin T, et al. Impaired autoregulation in preterm infants identified by using spatially resolved spectroscopy. Pediatrics 2008;121:e604-11.

16. Alderliesten T, Lemmers PM, Smarius JJ, van de Vosse RE, Baerts W, van Bel F. Cerebral oxygenation, extraction, and autoregulation in very preterm infants who develop peri-intraventricular hemorrhage. J Pediatr 2013;162:698-704.e2.

17. Baerts W, van Bel F, Thewissen L, Derks JB, Lemmers PM. Tocolytic indomethacin: effects on neonatal haemodynamics and cerebral autoregulation in the preterm newborn. Arch Dis Child Fetal Neonatal Ed 2013;98: F419-23.

18. Ramaekers VT, Casaer P, Daniels H, Marchal G. The influence of blood transfusion on brain blood flow autoregulation among stable preterm infants. Early Hum Dev 1992;30:211-20.

19. Vanderhaegen J, Vanhaesebrouck S, Vanhole C, Casaer P, Naulaers G. The effect of glycaemia on the cerebral oxygenation in very low birthweight infants as measured by near-infrared spectroscopy. Adv Exp Med Biol 2010;662:461-6.

20. Pryds O, Greisen G, Lou H, Friis-Hansen B. Heterogeneity of cerebral vasoreactivity in preterm infants supported by mechanical ventilation. J Pediatr 1989;115:638-45.

21. Kaiser JR, Gauss CH, Williams DK. The effects of hypercapnia on cerebral autoregulation in ventilated very low birth weight infants. Pediatr Res 2005;58:931-5.

22. Fanaroff JM, Wilson-Costello DE, Newman NS, Montpetite MM, Fanaroff AA. Treated hypotension is associated with neonatal morbidity and hearing loss in extremely low birth weight infants. Pediatrics 2006;117:1131-5.

23. Kuint J, Barak M, Morag I, Maayan-Metzger A. Early treated hypotension and outcome in very low birth weight infants. Neonatology 2009;95:311-6.

24. Eriksen VR, Hahn GH, Greisen G. Dopamine therapy is associated with impaired cerebral autoregulation in preterm infants. Acta Paediatr 2014;103:1221-6.

25. Seri I, Rudas G, Bors Z, Kanyicska B, Tulassay T. Effects of low-dose dopamine infusion on cardiovascular and renal functions, cerebral blood flow, and plasma catecholamine levels in sick preterm neonates. Pediatr Res 1993;34:742-9.

26. Lundstrøm K, Pryds O, Greisen G. The haemodynamic effects of dopamine and volume expansion in sick preterm infants. Early Hum Dev 2000;57:157-63.

27. Munro MJ, Walker AM, Barfield CP. Hypotensive extremely low birth weight infants have reduced cerebral blood flow. Pediatrics 2004;114: 1591-6.

28. Walsh MC, Kliegman RM. Necrotizing enterocolitis: treatment based on staging criteria. Pediatr Clin North Am 1986;33:179-201.

29. Brazy JE, Lewis DV, Mitnick MH, Jöbsis vander Vliet FF. Noninvasive monitoring of cerebral oxygenation in preterm infants: preliminary observations. Pediatrics 1985;75:217-25.

30. Lemmers PM, Toet M, van Schelven LJ, van Bel F. Cerebral oxygenation and cerebral oxygen extraction in the preterm infant: the impact of respiratory distress syndrome. Exp Brain Res 2006;173:458-67.

31. Naulaers G, Meyns B, Miserez M, et al. Use of tissue oxygenation index and fractional tissue oxygen extraction as non-invasive parameters for cerebral oxygenation. A validation study in piglets. Neonatology 2007;92:120-6.

32. Schumann P, Touzani O, Young AR, Morello R, Baron JC, MacKenzie ET. Evaluation of the ratio of cerebral blood flow to cerebral blood volume as an index of local cerebral perfusion pressure. Brain 1998;121 (Pt 7):1369-79. 